\title{
Investigation of catalytic glycolysis of polyethylene terephthalate by differential scanning calorimetry
}

\author{
C.Y. Kao ${ }^{\mathrm{a}}$, W.H. Cheng ${ }^{\mathrm{b}, *}$, B.Z. Wan ${ }^{\mathrm{a}}$ \\ ${ }^{a}$ Department of Chemical Engineering, National Taiwan University, Taipei, Taiwan \\ ${ }^{\mathrm{b}}$ Department of Chemical Engineering, Chang Gung College of Medicine and Technology, Kweishan, Taoyuan, Taiwan
}

Received 17 July 1996; accepted 12 December 1996

\begin{abstract}
The glycolysis of polyethylene terephthalate resin with excess amount of ethylene glycol, using metal acetates as catalysts, was examined by differential scanning calorimetry (DSC). The experiments were carried out under a nitrogen atmosphere of $600 \mathrm{psi}$. The efficiency of glycolysis was measured from the peak temperature associated with reaction endotherm. Among all the catalysts studied zinc acetate was confirmed to be the most effective for glycolysis. The glycolyzed products were mainly composed of small oligomers of bis-hydroxyethyl terephthalate in the presence of zinc acetate. Two isoconversion methods of kinetic analysis were applied for estimating the activation energy of glycolysis. The activation energy was lowered either by adding zinc acetate or by decreasing resin size. The results suggest that the depolymerization or the removal of polymer molecules from solid matrix should be the rate-limiting step of polyethylene terephthalate (PET) glycolysis, and that zinc acetate might facilitate the bond scission of polymer chains. (C) 1997 Elsevier Science B.V.
\end{abstract}

Keywords: Catalysis; Differential scanning calorimetry (DSC); Glycolysis; Kinetics; Polyethylene terephthalate (PET)

\section{Introduction}

Polyethylene terephthalate (PET) is an industrially important thermoplastic resin, which is extensively applied in the production of fibers, containers, and films. Many attempts are currently directed toward recycling of waste PET because of the interests in environmental protection and economic benefits [1]. Glycolysis is one of the effective methods for chemical recycling of PET. In this process, bis-hydroxyethyl

\footnotetext{
*Corresponding author. Tel.:(+886-3) 3283016; fax $(+886-3)$ 3283031 .
}

terephthalate (BHET) monomer and its oligomers are recovered from the waste in the presence of excess ethylene glycol (EG), as reported by Vaidya and Nadkarni [2,3]. Metal acetates, commonly used in the transesterification stage of PET production, are effective catalysts for glycolysis. Baliga and Wong [4] investigated some metal acetates $(\mathrm{Zn}, \mathrm{Pb}, \mathrm{Mn}, \mathrm{Co})$ for catalyzing glycolytic depolymerization of PET under reflux at $190^{\circ} \mathrm{C}$. Zinc acetate was found to be most active; however, the reaction mechanisms have not been clear. On the other hand, Chen et al. [5] studied the kinetics of PET glycolysis in a pressurized reactor at temperatures between 190 and $240^{\circ} \mathrm{C}$. They found that the glycolysis rate was second order to EG con- 
centration, and concluded that EG acted as both reactant and catalyst in the chain scission step. Nevertheless, their assertion contradicted the earlier and later findings of Challa and Campanelli et al. [6-9], which suggested that EG did not play a significant role as an internal catalyst in the glycolytic depolymerization of PET. Moreover, it was noted that zinc salts did not increase glycolysis rate at temperatures above $265^{\circ} \mathrm{C}$ [9], due to mass transfer limitation at high reaction temperature.

The techniques of thermal analysis have been widely used to characterize the properties of polymeric materials, but scarcely applied to investigate polymer reactions. Wolf et al. [10] first developed a method based on DSC to measure the catalytic activity of metal compounds for polycondensation of BHET to produce PET. Gamlen et al. [11] later improved this DSC method and developed thermogravimetric analysis (TGA) technique for the same purpose. Their observations of catalytic effectiveness agreed with the data from conventional methods; however, the thermal analysis techniques are more convenient. The application of DSC technique to investigate the glycolysis of PET has first been done by Johnson and Teeters [12]. Only manganese acetate was used as a catalyst in their study. The activation energy of glycolysis under high pressure was calculated by isothermal kinetic analysis in their work. However, it was much higher than those obtained from studies using conventional batch reactors [6-8]. The reasons for this discrepancy were still not clear.

In the present work, DSC was employed for investigating the glycolysis of PET with excess amount of EG in the presence of various metal acetate catalysts. The catalytic activity and the extent of reaction were obtained from the scanning thermograms. Furthermore, two isoconversion methods of kinetic analysis were used to study the effect of the zinc acetate catalyst and the PET resin size on the activation energy of glycolysis.c

\section{Experimental}

PET powder was prepared by grinding chips of fiber grade, which were supplied by Nan Ya Plastic Co., Taiwan. It was subsequently sieved to obtain the particle size of $45 / 70$ mesh for regular testing. Smaller powder of 70/120 mesh was also obtained for examining the size effect. The intrinsic viscosity (IV) of PET used in this study was measured in a $6: 4(\mathrm{w} / \mathrm{w})$ phenol/1,1,2,2-tetrachloroethane solution at $30^{\circ} \mathrm{C}$. The IV value of $0.60 \mathrm{dl} \mathrm{g}^{-1}$ corresponding to the average molecular weight (M) of 17000 , was calculated from the equation of IV $=7.55 \times 10^{-4} \mathrm{M}^{0.685}$ [13]. The melting of this PET material yielded an endotherm about $40 \mathrm{~J} \mathrm{~g}^{-1}$, over the temperature range of 245 to $260^{\circ} \mathrm{C}$, as determined by a DSC at a scanning rate of $10^{\circ} \mathrm{C} \mathrm{min}{ }^{-1}$.

Five metal acetates were employed as catalysts: cobalt acetate, $\mathrm{Co}\left(\mathrm{CH}_{3} \mathrm{COO}\right)_{2} \cdot 4 \mathrm{H}_{2} \mathrm{O}$; cupric acetate, $\mathrm{Cu}\left(\mathrm{CH}_{3} \mathrm{COO}\right)_{2} \cdot \mathrm{H}_{2} \mathrm{O} ;$ manganese acetate, $\mathrm{Mn}\left(\mathrm{CH}_{3} \mathrm{COO}\right)_{2} \cdot 4 \mathrm{H}_{2} \mathrm{O}$; sodium acetate, $\mathrm{Na}\left(\mathrm{CH}_{3-}\right.$ $\mathrm{COO}) \cdot 3 \mathrm{H}_{2} \mathrm{O}$; and zinc acetate, $\mathrm{Zn}\left(\mathrm{CH}_{3} \mathrm{COO}\right)_{2} \cdot 2 \mathrm{H}_{2} \mathrm{O}$. The metal acetate was preliminarily mixed with PET powder ( $45 / 70$ mesh) by incipient wetness impregnation. The mixture was then dried at $60^{\circ} \mathrm{C}$ for $12 \mathrm{~h}$. The metal loading of each sample was controlled at the level of $0.10 \mathrm{mmol}$ per gram of PET. The blank sample of PET was similarly prepared by the same procedures except that no catalyst was added.

The thermogram of each sample was measured on a DSC (Du Pont 910) equipped with a TA 2200 thermal analyzer. The sample was placed in a hermetically sealed aluminum pan. Another empty pan was used as the reference during measurements. The calibrations of DSC were made with standard indium and zinc metals.

The glycolysis of PET with EG in a fixed charge ratio of $2 \mathrm{mg}$ of PET to $4 \mathrm{mg}$ of EG was investigated with a pressurized DSC cell, under a nitrogen atmosphere of $600 \mathrm{psi}$. The boiling point of EG under this pressure was elevated to the temperature above $300^{\circ} \mathrm{C}$; therefore, the thermogram of reaction under $300^{\circ} \mathrm{C}$ could be precisely observed without interference from evaporation of EG. The pan with the tested sample was removed from the cell after the DSC scanning and pierced a hole of $1 \mathrm{~mm}$ in diameter on the center of upper lid. It was then placed in another DSC cell at one atmosphere. The sample was isothermally heated at $150^{\circ} \mathrm{C}$ for $30 \mathrm{~min}$ to evaporate all of the unreacted EG under a nitrogen flow rate of $100 \mathrm{ml} \mathrm{min}^{-1}$. The glycolyzed products following these treatments were examined with its melting thermogram from 30 to $300^{\circ} \mathrm{C}$. 


\section{Results and discussion}

\subsection{Catalytic effect on PET glycolysis}

The DSC thermograms of mixtures of PET and EG in the presence of various metal acetate catalysts under nitrogen at $600 \mathrm{psi}$ are shown in Fig. 1. The heating rates were maintained at $2.5^{\circ} \mathrm{C} \mathrm{min}^{-1}$. Only one endotherm, ranged from 210 to $230^{\circ} \mathrm{C}$, was observed during each scanning. The peak temperatures of these endotherms decreased in the presence of $\mathrm{Na}^{+}, \mathrm{Cu}^{2+}$, $\mathrm{Co}^{2+}, \mathrm{Mn}^{2+}$, and $\mathrm{Zn}^{2+}$. The endothermic peaks should correspond to the reaction heat generated by the glycolysis of PET with EG, because both endothermic temperatures of the evaporation of EG and the melting of PET resin are well above these peak temperatures. Thus, the location of the peak temperature can indicate the relative activity of glycolysis; in other words, the catalytic activity is higher if the peak temperature is lower. Zinc acetate, therefore, is the most effective catalyst for glycolysis among metal acetates investigated in this study; whereas, the least effective one is sodium acetate, whose peak temperature is close to that of the sample without catalyst. Moreover, the order of magnitude of catalytic activities in this work is consistent with that of the glycolysis reaction carried out in a bench-scale batch reactor [4].

The heat of glycolysis of PET melt to dimer or monomer of BHET was zero or $44 \mathrm{~J} \mathrm{~min}^{-1}$ PET, as calculated from the reverse reaction of polycondensation, reported by Challa [7]. However, the endothermic values of thermograms from glycolysis of solid PET, indicated in Fig. 1, were ranged from 40$70 \mathrm{~J} \mathrm{~min}^{-1}$ of PET. The heat of PET glycolysis from its solid state is higher than that from its molten state by about $40 \mathrm{~J} \mathrm{~g}^{-1}$, which is equivalent to the heat for the melting of solid PET. Although the reaction temperature in this work was well below the melting temperature of PET, more energy required for glycolysis during DSC measurement was due to the heat needed for the removal of the polymer molecules from the solid matrices to the liquid state during the course of depolymerization of solid PET.

\subsection{Distribution of glycolyzed products}

Because zinc acetate showed the highest catalytic activity among the catalysts used in this study, the DSC thermograms after the PET glycolysis, in the presence or the absence of zinc acetate, were studied

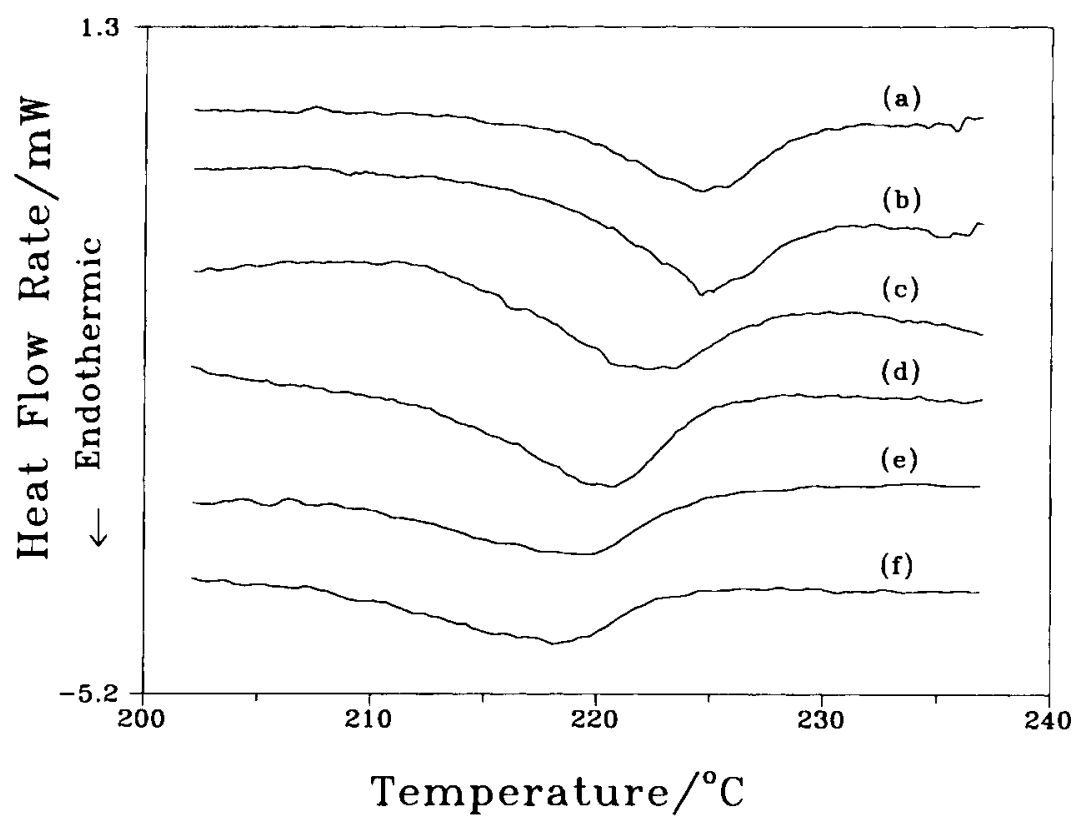

Fig. 1. DSC thermograms of mixtures of PET and EG $(w / w=2 / 1)$ in the presence of various metal acetates; heating rate is $2.5^{\circ} \mathrm{C} \mathrm{min}{ }^{-1}$ under 600 psi pressure. (a) No catalyst; (b) Sodium acetate; (c) Cupric acetate; (d) Cobalt acetate; (e) Manganese acetate; (f) Zinc acetate. 


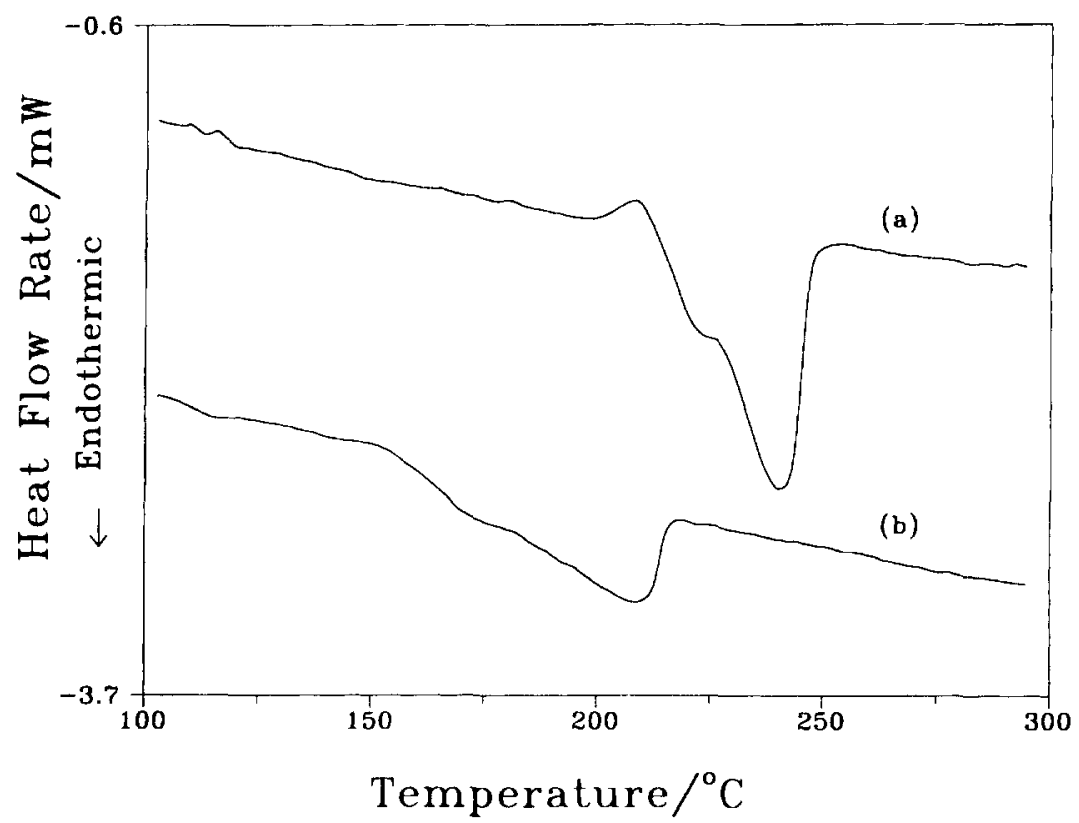

Fig. 2. DSC thermograms of mixtures after PET glycolysis in the presence or absence of zinc acetate; heating rate is $10^{\circ} \mathrm{C} \mathrm{min}{ }^{-1}$ under $100 \mathrm{ml} \mathrm{min}^{-1}$ nitrogen flow measured at one atmosphere. (a) No catalyst; (b) Zine acetate.

further. The experiments were carried out at one atmospheric pressure under $100 \mathrm{ml} \mathrm{min}{ }^{-1}$ nitrogen flow, following the removal of the unreacted EG under nitrogen flow at $150^{\circ} \mathrm{C}$ for $30 \mathrm{~min}$. The results are shown in Fig. 2. The endotherms corresponded to the melting of glycolyzed products. They were broad and overlapped with some neighboring peaks, which suggest the presence of different oligomers in the glycolyzed products. In the absence of catalysts, the melting of glycolyzed products occurred between 210 and $245^{\circ} \mathrm{C}$; on the other hand, the melting occurred between 160 and $210^{\circ} \mathrm{C}$, in the presence of zinc acetate catalyst. Using the relationship between molecular weight and melting point of oligomeric diols [14], we estimated that the average molecular weight of the glycolyzed products was larger than 1,000 in the absence of catalysts and was 450-830 when zinc acetate was added. The results demonstrate that with excess of ethylene glycol, PET of average molecular weight of 17000 can be depolymerized to oligomers to a high extent in the presence or absence of zinc acetate. However, zinc acetate can catalyze PET glycolysis and result in more complete depolymerization. Moreover, PET could not be depolymerized to BHET monomer or dimer in the DSC glycolysis system, but could be in the stirred liquid reactor system [4]. The difference should be due to the mass transfer limitation in the DSC system.

\subsection{Thermal degradation of PET}

In order to explore the effect of thermal degradation on PET glycolysis, thermal degradations of PET, in the presence or absence of metal acetates, were investigated. The influence of these catalysts on the melting endotherm of fresh PET was examined prior to the study of thermal degradation. It was found that the peaks corresponding to the melting endotherms were sharp and were about 256 or $257^{\circ} \mathrm{C}$, either in the presence or absence of metal acetates. Therefore, the existence of metal acetate in PET has no significant influence on melting. Furthermore, after the thermal degradation of PET at $280^{\circ} \mathrm{C}$ and one atmosphere of nitrogen for $30 \mathrm{~min}$, the melting thermograms of samples were measured and are shown in Fig. 3. It was found that the melting point of the sample without metal acetate was the same as that without any thermal treatment; thus, in the absence of catalysts, no 


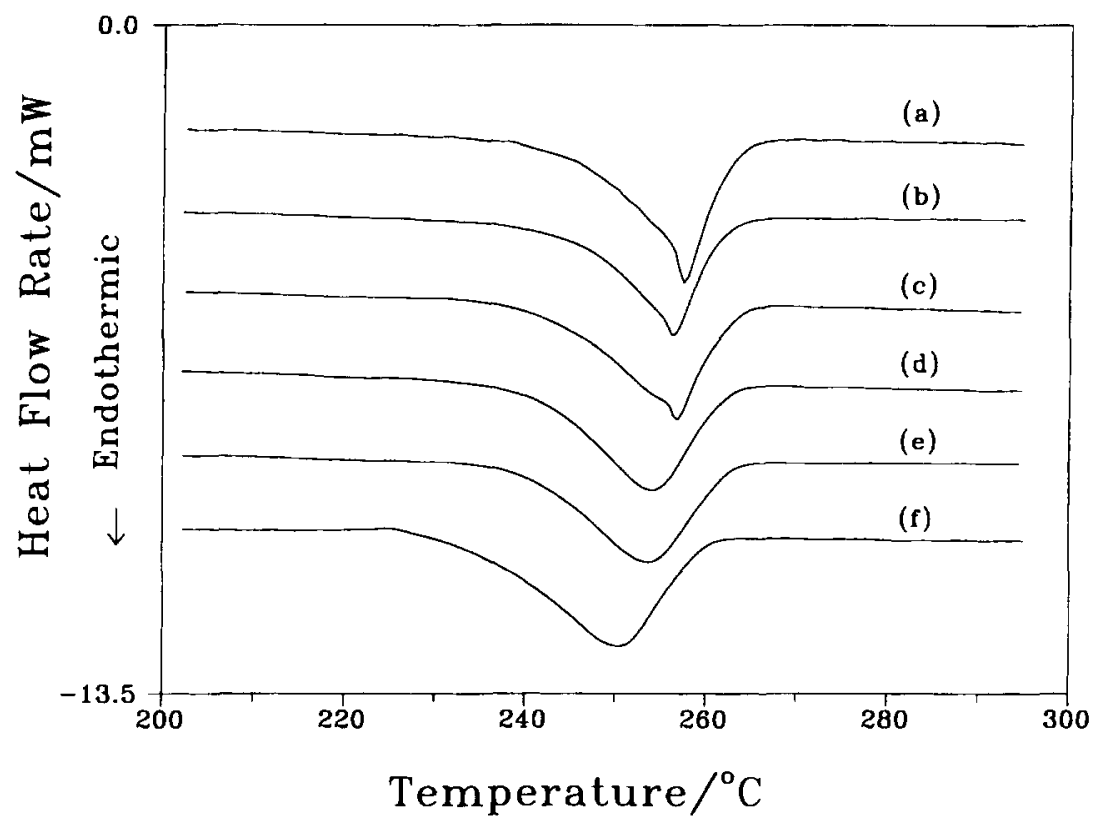

Fig. 3. DSC thermograms $\left(10^{\circ} \mathrm{C} \mathrm{min}{ }^{-1}\right.$ heating rate) of PET melting after thermal degradation in the presence of (a) no catalyst; (b) sodium acetate; (c) manganese acetate; (d) cobalt acetate; (e) cupric acetate; or (f) zinc acetate under one atmosphere of nitrogen at $280^{\circ} \mathrm{C}$ for 30 min.

significant degradation occurred on PET sample at $280^{\circ} \mathrm{C}$. Similarly, sodium acetate and manganese acetate possessed little activity for thermal degradation. In contrast, cobalt acetate, cupric acetate and zinc acetate did. The melting point of thermally degraded PET in the presence of the latter three catalysts shifted significantly to a lower temperature range, as compared with that of pure PET. Among them zinc acetate had the highest activity. The lower endothermic temperature indicates that the average molecular weight of PET should be reduced owing to the degradation of polymer chains. This phenomenon is consistent with the experimental results, reported by Zimmerman and Kim [15], that the decrease of solution viscosity and the increase of carboxyl group content were observed during thermal degradation. Furthermore, the reactivities of metal actates for thermal degradation (Fig. 3), are in agreement with their catalytic activities for glycolysis (Fig. 1) except manganese acetate. This suggests that the activity of metal acetates for degradation of polymer chain should be important to the catalysis of glycolysis. In other words, it is conceivable that some metal acetates, especially zinc acetate, might enhance the bond scission of polymer chains in
PET matrix during glycolysis, and therefore faciliates the reaction. Nevertheless, manganese acetate only catalyzes PET glycolysis without enhancing the bond scission. This may be the reason that manganese acetate possesses less activity for PET glycolysis than zinc acetate.

\subsection{Effect of PET size or DSC scanning rate on PET glycolysis}

Two different ranges of particle sizes of PET (45/70 and $70 / 120$ meshes) were used to investigate the size effect. Both of them possessed the same melting thermograms. Their thermograms of PET glycolysis are compared in Fig. 4. It can be observed that the endothermic peaks decreased to lower temperatures with the decrease of the particle size of PET, either in the presence or absence of zinc acetate. In other words, the smaller the size of PET is, the more easily PET depolymerization proceeds under excess of ethylene glycol. This is apparently due to the enhancement of mass transfer rate or depolymerization rate at the external surface of PET particles, where external mass transfer of ethylene glycol and PET glycolysis occur. 


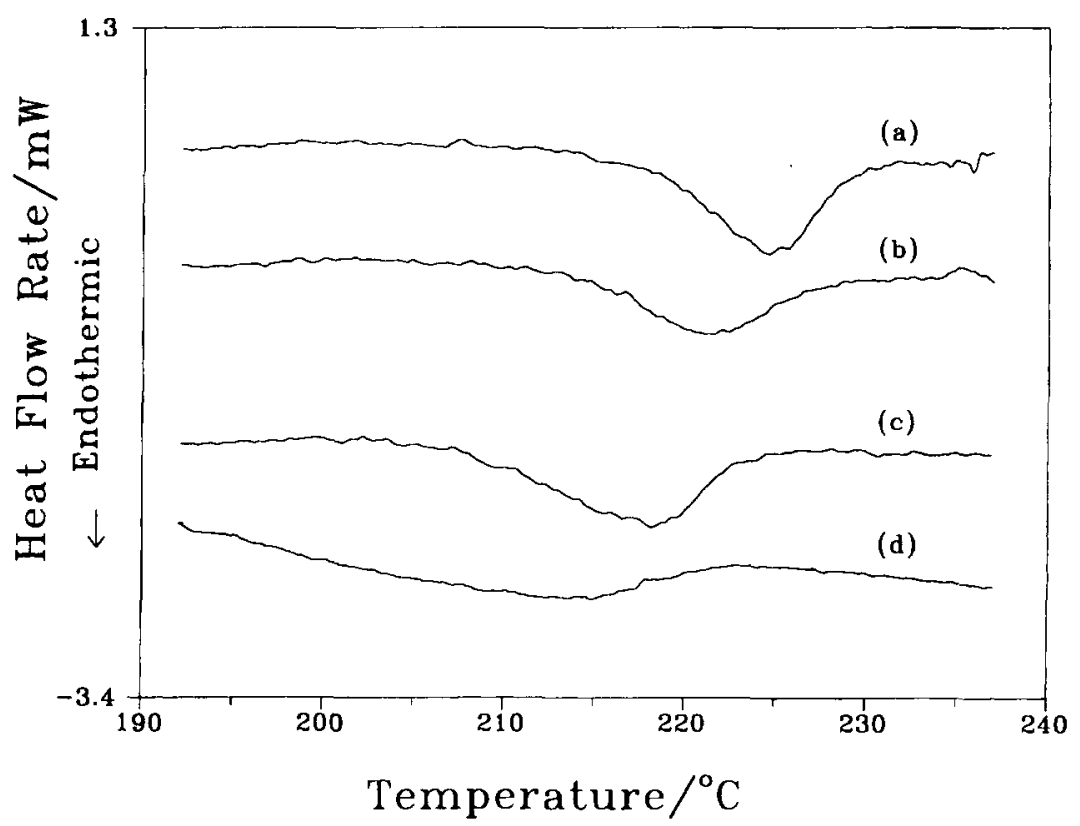

Fig. 4. Effect of resin sizes on DSC thermograms of PET glycolysis in the presence or absence of zinc acetate; heating rate is $2.5^{\circ} \mathrm{C}$ min ${ }^{-1}$ under nitrogen of 600 psi pressure. (a) No catalyst, resin size: $45 / 70$ mesh; (b) No catalyst, resin size: 70/120 mesh; (c) Zinc acetate, resin size: 45/70 mesh; (d) Zinc acetate, resin size: 70/120 mesh.

The smaller particles can provide larger surface area for mass transfer and for more contact between PET and ethylene glycol. Furthermore, Fig. 4 shows that the temperature for PET glycolysis in the presence of zinc acetate were dramatically lowered when PET particle size was reduced. It should be noted that the zinc acetate catalyst can only promote depolymerization reaction rate rather than mass transfer rate of ethylene glycol; in other words, the reaction rate is not controlled by the step of external mass transfer. The results suggest that depolymerization is a rate-determining step in our reaction system. Either the presence of zinc acetate or the increase of PET surface area for the reaction can enhance the rate of PET glycolysis.

The effect of DSC scanning rate, varying between 2.5 and $20^{\circ} \mathrm{c} \mathrm{min}^{-1}$, on the glycolysis under nitrogen of 600 psi pressure was explored. The thermograms in the absence and presence of zinc acetate are shown in Figs. 5 and 6. The peak temperatures of reaction endotherms in the absence of catalyst slightly increased with the heating rate; moreover, the trend was even more pronounced in the presence of zinc acetate. The marked elevation of peak temperature is a consequence of larger heat flux required for the endothermic reaction, which corresponds to more reaction proceeding in the same interval of time [16]. This demonstrates again that zinc acetate dramatically enhance the reaction rate of PET glycolysis

\subsection{Kinetic analysis}

Two kinds of isoconversion methods were employed for estimating the activation energy of glycolysis. Based on the first method developed by Ozawa [17], the activation energy was calculated from the slope of the logarithm of heating rate versus the reciprocal of absolute temperature at a given conversion. Based on the second method developed by Kissinger [18], the activation energy was calculated by the slope of the logarithm of heating rate divided by the square of peak temperature versus the reciprocal of peak temperature. The DSC data from Fig. 5 and Fig. 6 were analyzed by these two kinetic methods; whereas, the linear regression was carried out and the least square method was applied. The estimated values of activation energy for glycolysis are summarized in 


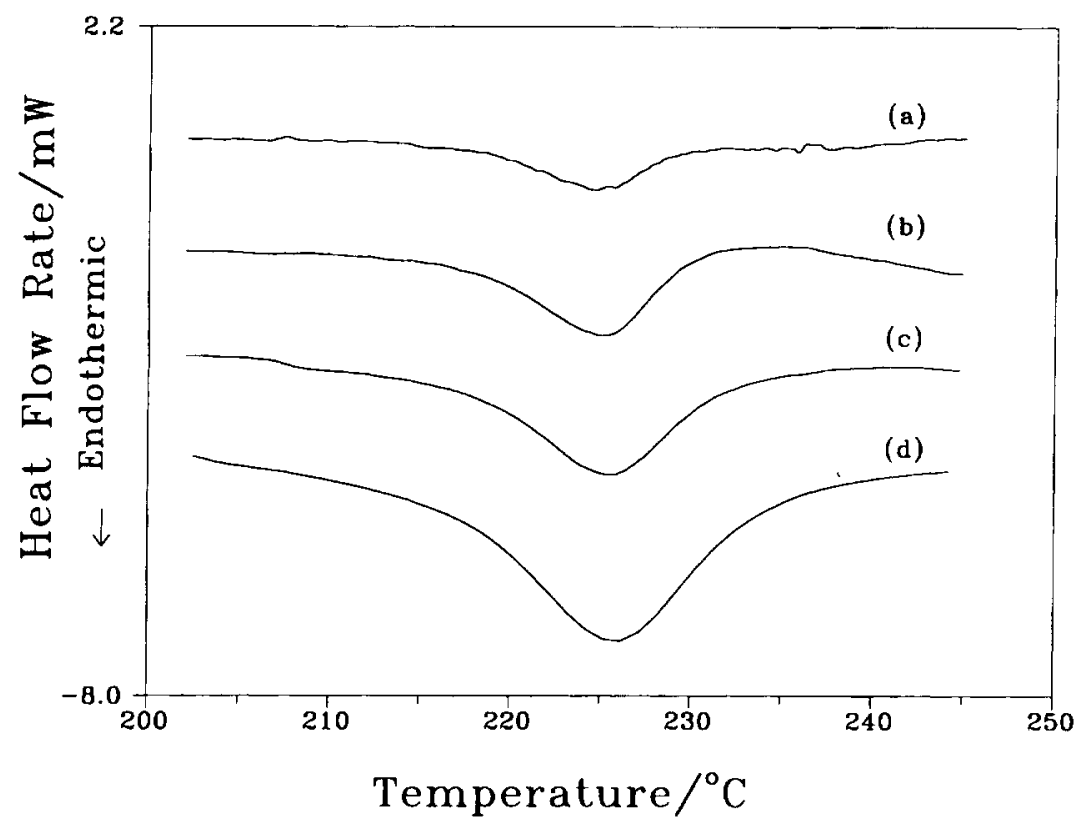

Fig. 5. Effect of scanning rate on DSC thermograms of PET glycolysis in the absence of catalyst. (a) $2.5^{\circ} \mathrm{C} \min ^{-1}$; (b) $5^{\circ} \mathrm{C} \mathrm{min}^{-1}$; (c) $10^{\circ} \mathrm{C}$ $\min ^{-1}$; (d) $20^{\circ} \mathrm{C} \min ^{-1}$.

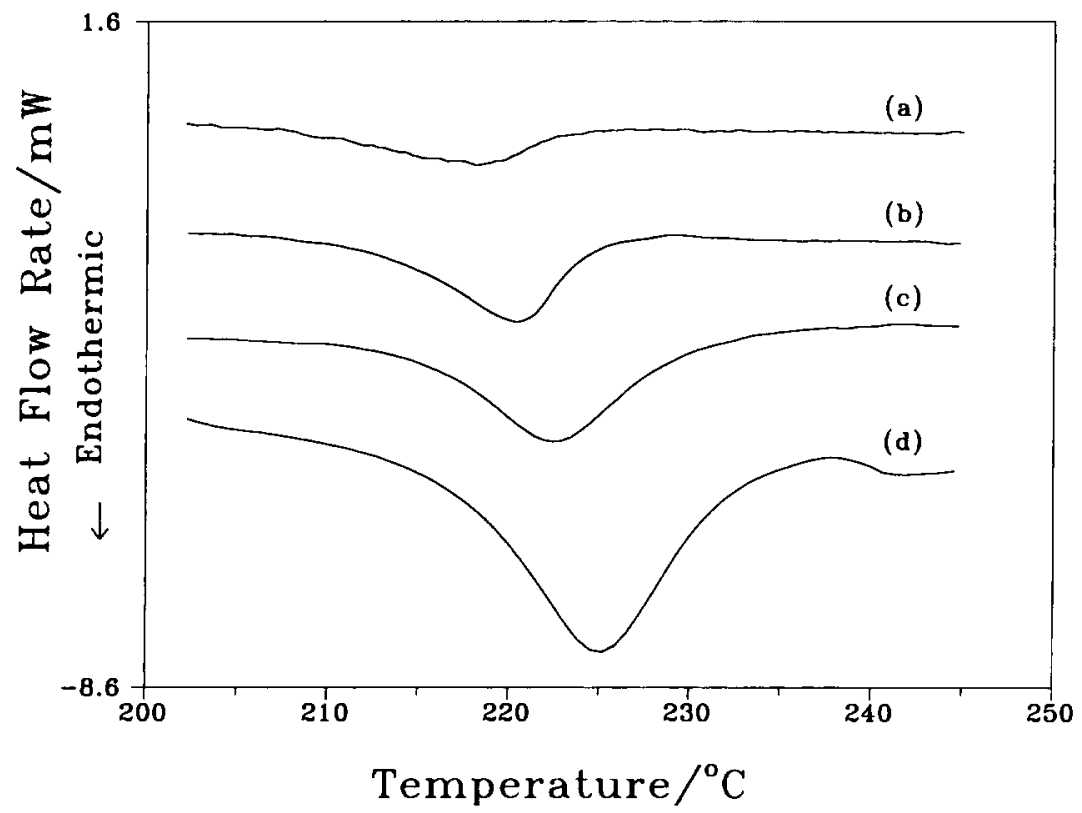

Fig. 6. Effect of scanning rate on DSC thermograms of PET glycolysis in the presence of zinc acetate. (a) $2.5^{\circ} \mathrm{C} \min ^{-1}$; (b) $5^{\circ} \mathrm{C}$ min ${ }^{-1}$; (c) $10^{\circ} \mathrm{C} \min ^{-1}$; (d) $20^{\circ} \mathrm{C} \mathrm{min}^{-1}$. 
Table 1

Estimated values of activation energy for glycolysis of PET ${ }^{\text {a }}$

\begin{tabular}{llll}
\hline Method & Conversion $^{\mathrm{b}}$ & \multicolumn{2}{c}{ Activation energy $/\left(\mathrm{kJ} \mathrm{mol}^{-1}\right)$} \\
\cline { 3 - 4 } & & No catalyst & Zinc acetate \\
\hline Ozawa [17] & 0.1 & very large & 491 \\
& 0.3 & 5124 & 489 \\
& 0.5 & 2677 & 492 \\
& 0.7 & 1631 & 477 \\
& 0.9 & 937 & 411 \\
Kissinger [18] & & 2693 & 694 \\
\hline
\end{tabular}

${ }^{a}$ Resin size: $45 / 70$ mesh.

${ }^{\mathrm{b}}$ Conversion is defined as the fraction of the total peak area generated by reaction endotherm.

Table 1. The corresponding correlation coefficients of these regressions were in the value of $0.97-0.99$. It was found that the values of activation energy calculated by Ozawa method in the absence of catalyst decreased with increasing degree of conversion. This may be because the activation energy noted here is much larger than the limited value of activation energy applied in the approximation of the exponential integral, given by Doyle [19], for Ozawa method. On the other hand, the activation energy as function of conversion also indicates that different reaction mechanisms should occur during the increase of temperature. When the reaction extent of glycolysis was low, PET in its original solid state reacted with EG. But with increasing conversion, PET was gradually degraded into small oligomers, which may be dissolved in EG at the reaction temperature, and then the subsequent glycolysis reaction could be conducted in liquid state. Therefore, it is reasonable that the activation energy of PET glycolysis in the absence of catalyst would be much higher in low conversion than in high conversion. In contrast, the change in activation energy with conversion was not apparent for the glycolysis in the presence of zinc acetate. This indicates that scission of PET chains catalyzed by zinc acetate should be fast even in its solid state.

Although the extent of depolymerization are different between the glycolysis reactions of PET in the presence and absence of zinc acetate, the activation energies estimated by the isoconversion methods can still be quantitatively compared between the two cases. The results of kinetic analysis in Table 1 show that the activation energy of glycolysis is obviously lower in the presence of zinc acetate than in the absence of catalyst; in fact, the value for the former is approximately one fourth of that for the latter, as estimated by the second method. The lowering of activation energy by zinc acetate is in agreement with its high catalytic activity for PET glycolysis, as stated above. On the other hand, the estimated activation energy for PET glycolysis is consistent with the value calculated from the results of isothermal reaction rate by Johnson and Teeters [12]. However, both activation energies estimated from two kinds of DSC approach, isoconversion or isothermal reaction rate, are abnormally larger than the values for glycolysis of PET melts in a stirring batch reactor, which are reported to be around $100 \mathrm{~kJ} \mathrm{~mol}^{-1}$ [7-9]. There might be a few reasons causing the difference in the estimated values of activation energy for PET glycolysis. One likely reason would be the nonuniformity of the heat flux during dynamic temperature measurements in such a heterogeneous solid-liquid reaction. However, the result of obtaining high activation energy similarly as ours, in isothermal DSC measurements [12] which possessed uniform heat flux, indicates that the effect of heat flux should not be dominant. Furthermore, the effects of other mass transfer limitations on reaction kinetics could not be significant because the catalytic effect of zinc acetate is pronounced. Another would be because different depolymerization mechanisms occurred from PET solid resin and molten resin, i.e. the higher activation energy is required for glycolysis of PET from solid state than from molten state. It was noted that zinc acetate did not increase glycolysis rate above the temperature of PET melting [9], and the early findings for the hydrolytic depolymerization of PET also showed the similar results $[20,21]$. These indicate that the glycolysis rate of molten PET is high at high temperature, and the overall reaction rate might be controlled by mass transfer steps. Therefore, the activation energy obtained is in fact much lower than that controlled by reaction step. On the other hand, the activation energy for thermal degradation of PET melts in the temperature range of 270 to $300^{\circ} \mathrm{C}$ in a batch reactor have been found to be around $190 \mathrm{~kJ} \mathrm{~mol}^{-1}[15,22]$, and the value is decreased to $150 \mathrm{~kJ} \mathrm{~mol}^{-1}$ in the presence of zinc acetate [15]. Meanwhile the effect of mass transfer should not be important under those reaction conditions because of proper mixing and low conversion. These results 
suggest that if the reaction rate is not controlled by mass transfer steps, the activation energy for the degradation of PET would be high and could be lowered by addition of catalyst. Thus, it may be reasonable to expect the high activation energies for PET glycolysis in this work, because the extra energy was required to break chains of PET matrix in its solid state. However, it should be noted that the reverse reaction of PET glycolysis for high extent of depolymerization is neglected in the kinetic analysis in our work and in Johnson and Teeters' work. It would make the observed reaction rate of forward depolymerization lower, although the reaction conditions in this research were in excess of ethylene glycol and were far apart from the equilibrium state. Therefore, the values of activation energy for PET glycolysis obtained in this work may be in some extent of inaccuracy caused by the neglect of the backward polycondensation during the glycolysis of PET.

The effect of particle size on the activation energy of glycolysis was also investigated. It can be observed in Table 2, the activation energy for glycolysis is reduced by $40-50 \%$ as the PET size decreases by about $40 \%$ when compared with the results listed in Table 1. Van Dooren and Müller [23] studied the effect of particle size on the activation energy of solid reactions determined by the calculation procedures of Ozawa and Kissinger. They found that the plot of Ozawa for the degradation of sodium bicarbonate gave activation energy of $105-113 \mathrm{~kJ} \mathrm{~mol}^{-1}$ for different size spectra. The slight increase in activation energies could partly be explained by the more predominant thermal resistance in larger particles. However, no significant effect of particle size on activation energy

Table 2

Estimated values of activation energy for glycolysis with smaller resin $^{\mathrm{a}}$

\begin{tabular}{llll}
\hline \multirow{2}{*}{ Method } & & \multicolumn{2}{l}{ Activation energy $/\left(\mathrm{kJ} \mathrm{mol}^{-1}\right)$} \\
\cline { 3 - 4 } & Conversion & No catalyst & Zinc acetate \\
\hline Ozawa [17] & 0.1 & 1921 & 203 \\
& 0.3 & 1723 & 230 \\
& 0.5 & 1349 & 258 \\
& 0.7 & 1067 & 287 \\
& 0.9 & 753 & 319 \\
Kissinger [18] & & 1969 & 314 \\
\hline
\end{tabular}

${ }^{\mathrm{a}}$ Resin size: $70 / 120$ mesh. was observed for the other cases under their investigation, even for that the mean activation energy of the solid-solid transitions was as high as $1765 \mathrm{~kJ} \mathrm{~mol}^{-1}$ for potassium nitrate or $1860 \mathrm{~kJ} \mathrm{~mol}^{-1}$ for hexamethylbenzene. It could thus be concluded from their results that the physical factor owing to particle size would not influence the measurement of the isoconversion DSC kinetics. Therefore, the apparent reduction of activation energy by decreasing the particle size of PET in this work is not an instrumental effect, which also strongly suggest that the reaction rate of glycolysis of solid PET could not be entirely controlled by the depolymerization step. The depolymerization rate would become comparable to the rate of mass transfer, such as the removal of polymer molecules from solid PET matrix, when the reaction rate was enhanced by decreasing PET resin size. Therefore, the apparent activation energy of PET glycolysis was reduced. In other words, the bond scission and the movement of polymer chains play the important roles for glycolysis below the temperature of PET melting.

\section{Conclusions}

The glycolysis of PET with ethylene glycol is studied by DSC technique and its efficiency can be identified by peak temperature of reaction endotherm. Zinc acetate was found to be the most effective catalyst among metal acetates examined. It also increased the extent of depolymerization so that the glycolytic products of smaller oligomers were obtained. The kinetic analysis showed that activation energy of glycolysis was decreased either by adding zinc acetate or by reducing PET resin size. Either the depolymerization or the removal of polymer molecules from solid matrix was suggested to be the rate-determining step of PET glycolysis. Zinc acetate might facilitate the bond scission of polymer chains and subsequently enhance the depolymerization rate.

\section{Acknowledgements}

The authors would like to thank Mr. S.C. Kao for his help in the DSC work. The financial support for this study from the National Science Council of Taiwan, 
R.O.C., through Grant No. NSC-84-2214-E-182-001 is gratefuly acknowledged.

\section{References}

[1] M.M. Nir, J. Miltz and A. Ram, Plastics Engineering, 49 (1993) 75 .

[2] U.R. Vaidya and V.M. Nadkarni, J. Appl. Polym. Sci., 35 (1988) 775.

[3] U.R. Vaidya and V.M. Nadkarni, J. Appl. Polym. Sci., 38 (1989) 1179 .

[4] S. Baliga and W.T. Wong, J. Polym. Sci. Part A. Polymer Chem., 27 (1989) 2071.

[5] J.Y. Chen, C.F. Ou, Y.C. Hu and C.C. Lin, J. Appl. Polym. Sci., 42 (1991) 1501.

[6] G. Challa, Makromol. Chem., 38 (1960) 105.

[7] G. Challa, Makromol. Chem., 38 (1960) 123.

[8] G. Challa, Makromol. Chem., 38 (1960) 138.

[9] J.R. Campanelli, M.R. Kamal and D.G. Cooper, J. Appl. Polym. Sci., 54 (1994) 1731.

[10] K.H. Wolf, B. Kuster, H. Herlinger, C.J. Tschang and E. Schollmeyer, Angew. Makromol. Chem., 68 (1978) 23.
[11] G.A. Gamlen, T.H. Shah, J.I. Bhatty and D. Dollimore, Thermochim. Acta, 106 (1986) 105.

[12] P.L. Johnson and D. Teeters, Polym. Prep., 32 (1991) 144.

[13] H. Kamatani, S. Konagaya and S. Nakamura, Polym. J., 12 (1980) 125.

[14] J. Brandrup and E.H. Immergut, Polymer Handbook, 3rd edn. (1967) p. VII-41.

[15] H. Zimmermman and N.T. Kim, Polym. Eng. Sci., 20 (1980) 680.

[16] W.W. Wendlandt, Thermal Analysis, Wiley-Interscience, New York, 3rd edn. (1986) Chap. 5.

[17] T. Ozawa, Bull. Chem. Soc. Jpn., 38 (1965) 1881.

[18] H.E. Kissinger, Anal. Chem., 29 (1957) 1702.

[19] C.D. Doyle, J. Appl. Polym. Sci., 6 (1962) 639.

[20] J.R. Campanelli, M.R. Kamal and D.G. Cooper, J. Appl. Polym. Sci., 48 (1993) 443.

[21] J.R. Campanelli, D.G. Cooper and M.R. Kamal, J. Appl. Polym. Sci., 53 (1994) 985.

[22] W.L. Hergenrother, J. Polym. Sci., Polym. Chem. Ed., 12 (1974) 875.

[23] A.A. van Dooren and B.W. Müller, Thermochim. Acta, 65 (1983) 257. 